\title{
Running of high patient volume radiation oncology department during COVID-19 crisis in India: our institutional strategy
}

\author{
Manoj Gupta, Rachit Ahuja, Sweety Gupta, Deepa Joseph, Rajesh Pasricha, Swati Verma, Laxman Pandey \\ Department of Radiation Oncology, All India Institute of Medical Science, Rishikesh, India
}

Received: April 4, 2020

Revised: April 28, 2020

Accepted: May 15, 2020

Correspondence:

Sweety Gupta,

Department of Radiation Oncology,

All India Institute of Medical Science,

Rishikesh, India.

Tel: +91-9891981332

E-mail: sweety.rt@aiimsrishikesh.edu. in ORCID:

https://orcid.org/0000-0002-3893-2781
Purpose: Due to COVID 19 pandemic, the treatment of cancer patients has become a dilemma for every oncologist. Cancer patients are at an increased risk of immunosuppression and have a higher risk to acquire any infection. There are individual experiences from some centers regarding the management of cancer patients during such a crisis. So we have developed our institutional strategy to balance between COVID and cancer management.

Materials and Methods: Radiation Oncology departmental meeting was held to prepare a consensus document on Radiotherapy schedules and department functioning during this pandemic.

Results: Strategies were taken in form of following areas were steps need to be taken to decrease risk of infection, categorise treatment on the basis of priority, radiotherapy schedules modification, academic meetings and management of COVID positive patient/personnel in Radiation Oncology department.

Conclusion: We hope to strike the balance in overcoming both the battles and emerge as winners. Stringent long term follow up will be done for assessing the response or any unforeseen treatment related sequelae.

Keywords: COVID-19, Malignancy, Radiotherapy, Immunity

\section{Introduction}

Coronaviruses are enveloped, single-stranded, positive-strand RNA viruses and are classified under Nidovirales. The 2019-novel coronavirus (2019-nCoV) is classified as a novel betacoronavirus belonging to the sarbecovirus subgenus of Coronaviridae family [1-3]. The 2019-nCoV is the third coronavirus to exhibit cross species infection from animals to humans. The previous two being 2002 outbreak caused by the severe acute respiratory syndrome coronavirus (SARS-CoV) and the 2012 outbreak caused by the Middle East respiratory syndrome coronavirus (MERS-CoV) $[4,5]$. Coronaviruses are known to cause common colds in human adults and children. It typically causes common cold like symptoms in immunocompetent individuals. Coronavirus disease 2019 (COVID-19) has a unique pathogenicity with its ability to cause both upper as well as lower respiratory tract infection
We intend to clarify the common doubts being faced by the high volume centres regarding the functioning of the department, the treatment of patients and safety of radiation personnel.

\section{How virulent is the COVID-19?}

According to the World Health Organization (WHO) seasonal flu has R0 at 1.3 whereas average contagion metric 2-2.5 which means that a person harbouring COVID-19 can transmit it to two people. However, this is less than SARS with RO of 4 and MERS RO of 2.5 to 7.2 [6]. In current scenario most important public measure remains the self-initiated quarantine of minimum 14 days for all the suspected patients with mild symptoms. All suspected patients and symptomatic contacts of all confirmed cases should be subjected to tests for confirmation [7]. 


\section{COVID-19 and its relevance to malignancy patients: why worry?}

SARS-CoV-2 a novel coronavirus first detected in Wuhan, China in December 2019 is the pathogen responsible for this current pandemic [7-9]. As per the official WHO website at the time of writing this article as on April 4, 2020 there are 1,098,006 cases and 59,141 deaths reported worldwide. Currently a whopping 205 countries and regions are in the grip of this pandemic along with two international conveyance. The United States and Europe have emerged as a new epicentre for COVID-19 with mortality and morbidity increasing daily. The United States and Italy are hit worst in current times with reported cases of 277,161 and death toll of 7,392 in the United States and 119,827 cases with 14,681 deaths reported in Italy. India currently has 2567 cases and 72 deaths [10]. Main measure to curb this pandemic is quarantine and social distancing. Everyone should stay at home to prevent community transmission. Thus, patients face difficulty in commuting to hospital. Radiotherapy being prolonged daily treatment needs attention during such crisis. Indian subcontinent has a population of over 1.35 billion and 1,157,294 new cases of malignancy are being diagnosed in a year as per GLOBOCAN 2018 [11], thus making treatment of malignancy as crucial as maintaining a strict vigil for COVID-19 patients.

\section{Why worry in cancer patients and care givers?}

Majority patients diagnosed of malignancy present in advanced stage disease are malnourished and have a weak innate immunity due to the disease process, poor nutritional status as well as the side effects of therapy (specially chemotherapy). Increased propensity of cancer patients of developing complications related to COVID-19 has also been seen by the Chinese data given by Liang et al. [12] reported a significantly higher incidence of severe events, i.e., death or ICU admission requiring invasive ventilation among individuals with a cancer history than those without (39\% vs. 8\%; $p=0.0003$ ), in 2007 Chinese patients hospitalized with COVID-19. Although the sample size was small but it does serves as an indicator of the need of being cautious. On the other end of the spectrum are the healthcare providers who are constantly at an increased risk of exposure for acquiring the infection.

\section{Materials and Methods}

On Saturday, March 21, 2020 in the wake of crisis, radiation oncology departmental meeting was held to prepare an expert consensus that will serve as a strategy in this time of crisis. The conversation was based on how to reduce transmission, manage decreased workforce and continue treatment in the presence of infection. So strategy was defined to cover all the aspects of functioning radiation oncology department, treating patients and maintaining personal protection.

\section{Results}

Strategies are summarized in form of following areas were steps need to be taken: (1) decrease risk of infection, (2) categorise treatment on the basis of priority, (3) radiotherapy schedules modification, (4) academic meetings, and (5) management of COVID-19 positive patient/personnel in radiation oncology department.

\section{Decrease risk of infection}

The entire workforce of the department was called to the meeting and educated about the current situation with the risk involved and the need of continuing our essential services in this time. The healthcare providers are divided into two risk groups as per patient interaction (Fig. 1)

\section{1) General measures for decreasing risk of workforce}

Clean waiting area with adequate distancing between the waiting benches and the patients. Time slots defined for patients and treating no more than 5 patients per hour. Stringent use mask and follow standard hand hygiene practices using alcohol-based hand sanitizers and soap and water. Organised quick practical session for the departmental staff on correct use of personal protective gear, their disposal and standard practice of hand hygiene conducted. Patients on treatment should be scheduled in time slots to decrease patient waiting and contact. Detailed travel history to COVID-19 infected area or close contact with a person infected with coronavirus. Thermal screening of all patients/staff at radiation premises to be carried out regularly. In case any suspected patients, he/she will to be sent to screening outpatient department (OPD) and handled by department of medicine/emergency as per institutional policy. Staff divided into two groups and postings to be done in weekly shifts separated by time and location. The departmental staff has been strategically divided into two halves having one team at work and one in back up. Thus, ensuring smooth functioning of the department as well as having workforce in backup in case some personnel is exposed/suspected. CT-simulation have been dispersed throughout the day to minimize the number of people in the waiting rooms.

\section{2) Minimize personal contact}

Time: minimalize patient contact time;

Distance: minimum $1 \mathrm{~m}$;

Shielding: adequate personal protective equipment (mask/scrubs). 


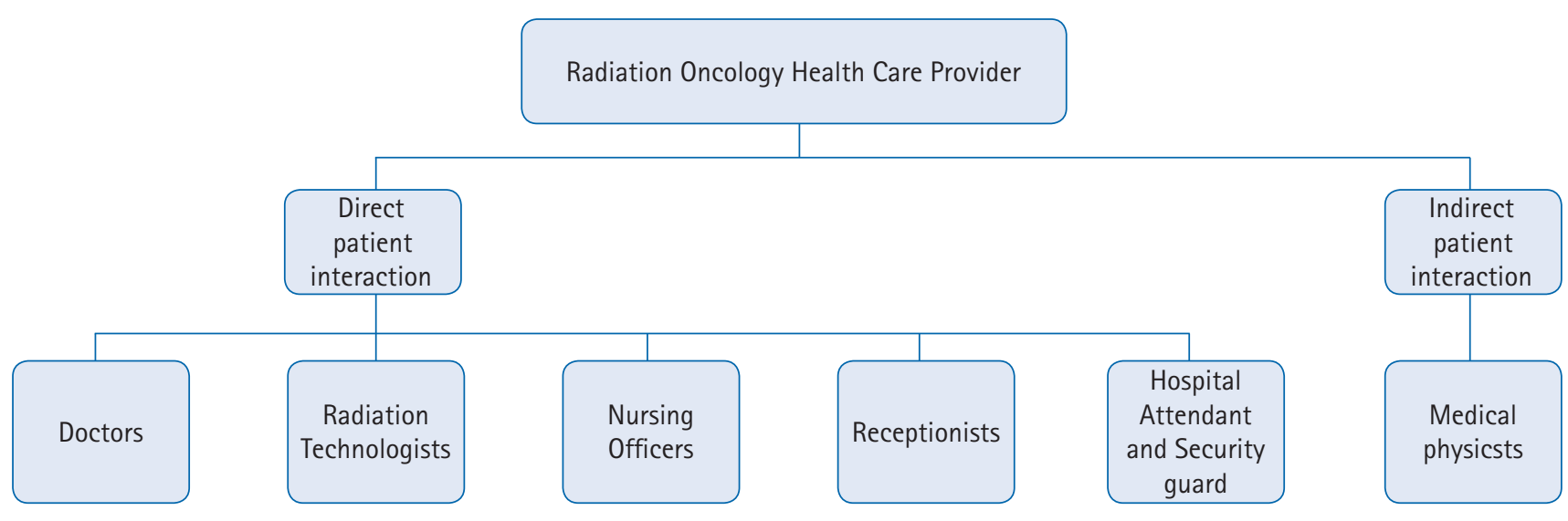

Fig. 1. Healthcare providers division into two risk groups as per patient interaction.

Telemedicine consultation have been started to minimize patients visits. But strictly no prescription of intravenous medication to be prescribed telephonically. Patients to be counselled mainly for symptomatic care /continuation of any metronomic therapy patient was already receiving. Postponement of elective post treatment follow up to be done.

\section{3) Directive to patients}

All the patients visiting radiation oncology wing are to be advised to follow social distancing at least $1 \mathrm{~m} / 3$ feet distance between each other. Stick to the time schedule allotted to each patient. They have report any new onset fever cold cough or breathing difficulty. Only one attendant should accompany if necessary and avoid bringing young children or old people as attendant to the hospital. They are also advised to maintain good diet to boost immunity. Patients are suggested to visit hospital only for urgent medical care. Visit to crowded areas to be avoided. They are educated to wash hands frequently with a soap/sanitizer. Elderly patients especially with symptoms are at highest risk and extra precaution should be taken.

\section{4) Outpatient department consultation}

Consultants and residents are instructed to maintain at least one-meter distance with care giver and patient. Take detailed history of recent travel to COVID-19 infected area or close contact with a person infected with coronavirus, check for any symptoms of viral infection in all patients. New patients enrolment to be done with quick short case history and examination to decrease contact time. Unnecessary repeated multiple examination of patients should be avoided.

\section{5) Inpatient department admissions}

In view of high risk of malignancy patients acquiring COVID-19, minimal inpatient department (IPD) admissions to be done. Also, majority beds will be kept vacant as a backup in case of any crisis

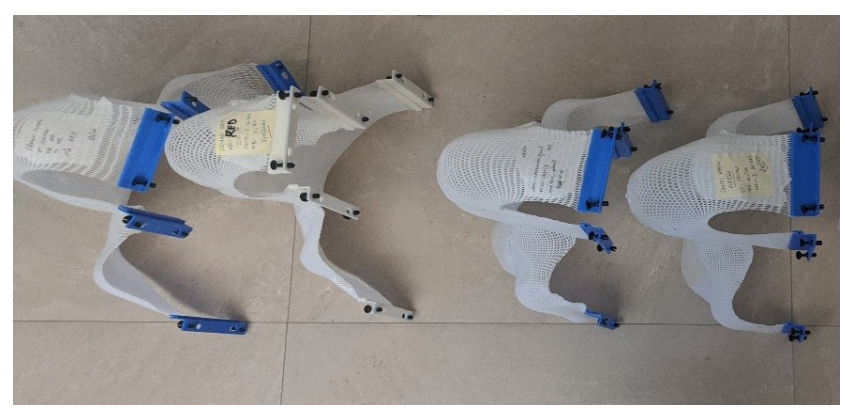

Fig. 2. The correct placement of immobilisation devices.

of COVID-19 outbreak occurs in the region. Full precautions would be maintained for assuring the safety of hospital personnel as well as patient to decrease possibility of cross infection. Neutropenic patients would be admitted in the IPD and discharged only after full recovery. Review with infectious diseases team would be taken if any symptom suggestive of COVID-19.

\section{6) Radiation oncology staff education}

Use of proper personal protection equipment (PPE) by the department staff. Regular training and stringent practice of hand hygiene. Sanitisation of (1) immobilisation casts/devices: once a day with chlorite-based solution. Also, casts to be kept separately and not stacked over one another (Fig. 2); (2) treatment/simulation couch: before starting treatment for the day and after treatment of each patient hourly; (3) door handles and knobs every 3 hourly; and (4) sheets/linen used for patients to be washed and sterilised.

\section{Categorise treatment on the basis of priority}

(1) Priority high: Patients undergoing radiation with radical intent for cure in which delay in treatment onset may jeopardize outcome.

(2) Priority moderate: Radical radiotherapy for less aggressive tu- 
mours or urgent palliative radiotherapy like in patients with malignant spinal cord compression to avoid neurological damage.

(3) Priority low: Radiation in an adjuvant setting where a complete resection of disease with good margins and less than 15\%-20\% risk of recurrence over 10 years, and patients with less aggressive tumours where radiation is a part of treatment protocol but can be postponed owing to low risk category and slow growth of tumour.

\section{Radiotherapy schedules modification}

Evidence-based of possible hypofractionated regimens or observational strategies for a variety of entities which can be considered during this crisis. The potential benefits and risks of altered fractionations to be carefully discussed with the patient.

Hypofractionation rationale and importance in today's scenario are (1) biologically equivalent hypofractionated treatment regimens to be used; (2) helps minimize number of individual patient visits to department; (3) also helps decrease the total number of patients being treated in a day though still allowing us to keep the total patients treated the same; and (4) avoids delay of treatment in an already high-volume centre with a long waiting period.

Dose to critical organs-at-risk (OARs) would be respected and calculated as per LO model. Minimal use of radiation accessories involving physical contact. If any accessory if contaminated should be sent for testing and decontamination. Postoperative cases or cases where the addition of radiation/chemotherapy would not translate into much clinical benefit (elective low-grade including glioma, low risk tumours like breast and prostate) will be deferred for treatment. Any patient who tests positive for COVID-19 will not be treated till patient is asymptomatic and negative titres are achieved.

1) Site wise proposed radiotherapy schedule modifications in the time of COVID-19 crisis

Head and neck malignancies - need and rationale for changing the protocol: Our centre has a high burden of advanced stage III/IV head and neck malignancies requiring radical radiation for treatment. Any new patient being planned will be taken up for hypofractionated radiation. Alternate day treatment at higher dose per fraction will be considered without compromising oncological outcome. This will decrease hospital visits of patients at daily basis however completing the treatment in the same duration.

\section{2) Eligible patients}

Young patients, the Karnofsky performance scale (KPS) > 80, good expected clinical outcome is being considered for this regimen, dose of OARs specifically spinal cord ALARA (as low as reasonably achievable) not exceeding $40 \mathrm{~Gy}$, and no concurrent chemotherapy will be given to these patients. Proposed hypofractionated radio-
Table 1. Hypofractionated radiotherapy schedule for head and neck cancer with EQD2 and BED

\begin{tabular}{lccc}
\hline & High risk & Intermediate risk & Low risk \\
\hline Dose (Gy/fraction) & 3 & 2.65 & 2.34 \\
Total (Gy) & 63 & 55.65 & 49.14 \\
EQD2 & 68.25 & 58.6 & 50.5 \\
BED & 82 & 70.4 & 60.6
\end{tabular}

EQD2, equivalent dose in 2 Gy per fraction; BED, biological effective dose.

therapy dose and schedule is shown in (Table 1 and 2).

\section{3) General instructions for chemotherapy and treating other sites} Hormone positive (ER/PR+) breast tumours to be considered for hormonal therapy. Triple negative breast cancer (TNBC) may be considered for capecitabine oral tablets delaying definitive chemo by $2-4$ weeks. Carcinoma prostate to be considered for androgen deprivation therapy (ADT) and radiotherapy to be delayed. Palliative chemotherapy either delay or change to least toxic oral metronomic chemotherapy (capecitabine/gefitinib/methotrexate/cyclophosphamide/etoposide). Carcinoma cervix patients to be continued on concurrent weekly chemotherapy. Patients with primary CNS (high grade) tumours having ECOG $>2$ to be considered for oral temozolomide therapy only. Oral bisphosphonates to be considered for bone metastasis. Neutropenic patients to be admitted and treated as per protocol (in such patients review with COVID-19 team to be taken). Less common malignancies of all other sites to be treated with a tailored case-based approach. Strategies defined here for common malignancies being encountered at our centre and should be used as per institute and regional necessity.

\section{4) Brachytherapy}

Carcinoma cervix patients to be taken up for brachytherapy as per schedule. However, all patients as a part of institutional policy will be subjected to COVID-19 testing and only after being reported negative will be taken up for the procedure, thus minimising the risk of any unforeseen exposure to the health personnel and also reducing the stress from the infrastructure. In procedure room, staff will be minimized to one oncologist, one anaesthetist, one nurse and one operation theatre (OT) attendant.

\section{Academic meetings}

Regular post-graduate teaching activities have been withholding and teaching is being done using online platforms. Also, multidisciplinary meet is being conducted by telephonic consultation and if required with minimum number of consultants maintaining social distance. 
Table 2. Radiotherapy schedules for sites of malignancy

\begin{tabular}{|c|c|c|c|c|}
\hline Series\# & Site & Total dose & $\begin{array}{c}\text { Dose } \\
\text { (Gy/per fraction) }\end{array}$ & Duration (wk) \\
\hline \multirow[t]{3}{*}{1} & Cervix & & & \\
\hline & External radiotherapy & $45 \mathrm{~Gy}$ in $20 \mathrm{fx}$ & 2.25 & 4 \\
\hline & Brachytherapy & $\begin{array}{l}\text { Two sessions of } 9 \text { Gy each delivered } 1 \\
\text { week apart }\end{array}$ & & \\
\hline 2 & Brain primaries (high grade) & 40 Gy in $15 \mathrm{fx}$ & 2.67 & 3 \\
\hline \multirow[t]{3}{*}{3} & Rectum & & & \\
\hline & Preoperative, short-course (preferred) & $25 \mathrm{~Gy}$ in $5 \mathrm{fx}$ & 5 & 1 \\
\hline & Postoperative & $45 \mathrm{~Gy}$ in $20 \mathrm{fx}$ & 2.25 & 4 \\
\hline \multirow[t]{2}{*}{4} & Head and neck & & & \\
\hline & Postoperative & $55 \mathrm{~Gy}$ in $25 \mathrm{fx}$ & 2.25 & 5 \\
\hline \multirow[t]{4}{*}{5} & Breast & & & \\
\hline & Whole breast only & $26 \mathrm{~Gy}$ & 5.2 & One \# per week $\times 5$ wk \\
\hline & Chest wall onlya) & $26 \mathrm{~Gy}$ & 5.2 & One \# per week $\times 5$ wk \\
\hline & Nodal irradiation needed & $40 \mathrm{~Gy}$ in $15 \mathrm{fx}$ & 2.67 & 3 \\
\hline \multirow[t]{3}{*}{6} & Palliative radiation & & & \\
\hline & $\begin{array}{l}\text { Painful bone metastasis; Metastatic spinal cord } \\
\text { compression < } 48 \mathrm{hr} \text {; Symptomatic brain metas- } \\
\text { tasis; Tumour bleed }\end{array}$ & $8 \mathrm{~Gy}$ & 8 & SFRT \\
\hline & SVCO (symptomatic patients only) & $20 \mathrm{~Gy}$ in $5 \mathrm{fx}$ & 4 & 1 \\
\hline
\end{tabular}

SFRT, single fraction radiotherapy; SVCO, superior vena cava obstruction.

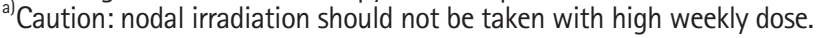

\section{Management of COVID-19 positive patient/personnel in radiation oncology department}

Any patient having a high clinical suspicion of being COVID-19 positive and a travel history form a hotspot region of COVID-19 cases would be taken for radiation or chemo only after being tested negative. In case a patient already on treatment tests positive, all the radiation personnel who had been in direct contact would be put under home quarantine and then subject to testing for COVID-19. The facility would be sealed and sanitisation would be done for the treatment unit the restroom and a thorough testing would be taken specially for the patients who were being treated. The backup team will be brought to the front for working with stringent use of PPE and sanitisation practices will be done.

\section{Discussion and Conclusion}

At this time of crisis world over radiation oncology departments need to take adequate precautions to reduce the likelihood of COVID-19 transmission. In oncology patients, it is a tricky situation and risk benefit calculation is very dicey because of risk of acquiring infection and at the same time possibility of spread of cancer. So we need to balance the situation in this scenario. Basic and universal precautions to be taken by staff before, during, and after each patient. Social distancing should be practised strictly. Staffing should be managed and work from home should be done in case it is possible so that in case any healthcare worker $(\mathrm{HCW})$ acquires infection or is quarantined another HCW can take care of work and department as whole is not shut down. All patients should wear protective masks during their stay in the radiotherapy department.

In the current times, an unforeseen pandemic rapidly gripping the world in its claws it is the responsibility of entire medical fraternity to tackle the new age pathogen without compromising the treatment and outcome of patients dealing with other ailments specially malignancy. This must be done keeping full vigil for the safety of the medical paramedical and allied staff working in the department. These strategies are intended to serve as guide for the common questions being faced by various oncology institutes. Stringent longterm follow-up will be done for assessing the response or any unforeseen treatment related sequelae. It is a testing time for all of humanity and together we intend to come over all the adversities to the best of our knowledge and experience. We hope to strike the balance in overcoming both the battles and emerge as winners.

\section{Conflict of Interest}

No potential conflict of interest relevant to this article was reported.

\section{References}

1. World Health Organization. Statement on the meeting of the International Health Regulations (2005) Emergency Committee re- 
garding the outbreak of novel coronavirus (2019-nCoV) [Internet]. Geneva, Switzerland: World Health Organization; c2020 [cited 2020 May 15]. Available from: https://www.who.int/newsroom/detail/23-01-2020-statement-on-the-meeting-of-the-international-health-regulations-(2005)-emergency-committee-regarding-the-outbreak-of-novel-coronavirus-(2019-ncov).

2. Wang C, Horby PW, Hayden FG, Gao GF. A novel coronavirus outbreak of global health concern. Lancet 2020;395:470-3.

3. Perlman S. Another decade, another Coronavirus. N Engl J Med 2020;382:760-2.

4. Cui J, Li F, Shi ZL. Origin and evolution of pathogenic coronaviruses. Nat Rev Microbiol 2019;17:181-92.

5. de Wit E, van Doremalen N, Falzarano D, Munster VJ. SARS and MERS: recent insights into emerging coronaviruses. Nat Rev Microbiol 2016;14:523-34.

6. Zhao S, Lin Q, Ran J, et al. Preliminary estimation of the basic reproduction number of novel coronavirus (2019-nCoV) in China, from 2019 to 2020: A data-driven analysis in the early phase of the outbreak. Int J Infect Dis 2020;92:214-7.

7. World Health Organization. Key considerations for repatriation and quarantine of travellers in relation to the outbreak of novel coronavirus 2019-nCoV [Internet]. Geneva, Switzerland: World Health Organization; c2020 [cited 2020 May 15]. Available from: https://www.who.int/news-room/articles-detail/key-consider- ations-for-repatriation-and-quarantine-of-travellers-in-relation-to-the-outbreak-of-novel-coronavirus-2019-ncov.

8. World Health Organization. Report of the WHO-China Joint Mission on Coronavirus Disease 2019 (COVID-19) [Internet]. Geneva, Switzerland: World Health Organization; c2020 [cited 2020 May 15]. Available from: https://www.who.int/publications-detail/report-of-the-who-china-joint-mission-on-coronavirus-disease2019-(covid-19).

9. Centers for Disease Control and Prevention. Coronavirus disease 2019 (COVID-19) situation summary [Internet]. Atlanta, GA: Centers for Disease Control and Prevention; c2020 [cited May 15]. Available from: https://www.cdc.gov/coronavirus/2019-ncov/ cases-updates/summary.html.

10. World Health Organization. Novel Coronavirus - China [Internet]. Geneva, Switzerland: World Health Organization; c2020 [cited 2020 May 15]. Available from: https://www.who.int/csr/ don/12-january-2020-novel-coronavirus-china/en/.

11. Ferlay J, Colombet M, Soerjomataram I, et al. Estimating the global cancer incidence and mortality in 2018: GLOBOCAN sources and methods. Int J Cancer 2019;144:1941-53.

12. Liang $W$, Guan $W_{1}$ Chen $R$, et al. Cancer patients in SARS-CoV-2 infection: a nationwide analysis in China. Lancet Oncol 2020; 21:335-7. 